\title{
Two-Jet Inclusive Cross Sections in Heavy-Ion Collisions in the Perturbative QCD
}

\author{
M.A. Braun ${ }^{a}$, R.S. Kolevatov ${ }^{a}$, B. Vlahovic ${ }^{b}$ \\ ${ }^{a}$ S.Petersburg State University, Russia, \\ ${ }^{b}$ North Carolina Central University, NC, USA
}

\begin{abstract}
In the framework of perturbative QCD, double inclusive cross sections for high $p_{t}$ parton production in nucleus-nucleus collisions are computed with multiple rescattering taken into account. The induced long-range correlations between numbers of jets at forward and backward rapidities are found to reach $10 \div 20 \%$ for light nuclei at $\sqrt{s}=200 \mathrm{GeV} / \mathrm{c}$ and to be suppressed for heavy nuclei and LHC energies.
\end{abstract}

\section{INTRODUCTION}

Particle production in high-energy heavy ion collisions is now at the center of experimental efforts to discover the quark-gluon plasma 1, 2]. The observed particle spectra are the result of different mechanisms which are responsible for the creation of initial high- $p_{t}$ partons, their propagation and subsequent hadronization. (Experimentally the initially produced partons can be traced as jets of hadrons, so that in the following we shall often use the term 'jet' for the produced parton in the pure theoretical sense, neglecting all the subtleties related to its actual determination in the experiment.) To be able to see the formation of the quark-gluon plasma against the background of more conventional effects, such as as gluon emission due to bremsstrahlung and multiple hard collisions in the nuclear medium, one has to fully study the consequences of the latter. Jet quenching due to gluon emission has been studied in considerable detail (see e.g. [3, 4]). Also the effects of rescattering in single particle inclusive spectra initiated by the observation of [5] has been considered in a series of articles for multiple soft [6, 7] and multiple hard pQCD scatterings [8, 9, 10, 11, 12, 13]. This paper generalizes the study of multiple hard collisions to double inclusive cross-sections and the following long-range correlations between the secondaries. Observation of such correlations has always served a very precise instrument for analyzing the dynamics of the interaction.

Following the framework introduced in [11] we consider only relatively hard collisions which allow for the perturbative QCD approach. The colliding nuclei are assumed to contain a variable number of partons with initially small transverse momenta, which become large as a result of hard collisions between partons belonging to the projectile and target. The latter are assumed to move fast along the collision axis in the opposite directions. In the present work we restrict ourselves to the study of high $p_{t}$ parton production at rapidities well separated from central rapidity region. Here longitudinal momenta of partons in the final state are much larger than the transverse ones. So we neglect attenuation of the former during the collisions and assume them to be conserved throughout the nucleus-nucleus interaction in agreement with the standard Glauber treatment. We neglect intrinsic correlations between partons inside the colliding nuclei which correspond to nuclear shadowing and take the partonic distributions just as a product of such distributions for the nucleons smeared out with the standard nuclear profile functions. This implies that we correctly describe quantum evolution of these distributions except for effects coming from interaction of partons between different nucleons in the nucleus, which should be studied from the DGLAP 
evolution equation for the nucleus as a whole or, at very small $x$, in the framework of the BFKL evolution (e.g. in the Colour Glass Condensate approach, see [14] and references therein). Jet quenching and hard parton contents of the participants are some of other important phenomena to be taken into account in the full treatment of jet production. Thus the physical phenomenon we study is restricted to multiple hard elastic scattering between partons. Our study is then to be considered as a baseline calculation to be followed by inclusion of all the above-mentioned effects

Our formalism closely follows [11] to which paper we refer the reader for details. As in [11], our basic approximations in the study of multiple partonic collisions are 1) purely elastic partonic collisions with conservation of longitudinal momenta and 2 ) factorization of the $S$-matrix into the product of elementary partonic $S$ - matrices (the Glauber approximation). We shall also use the notations of [11]. To make our presentation more self-contained we reproduce some basic notations below.

The initial states of the colliding nuclei A and B are represented as a superposition of states with different number of partons, having specific values of scaling variables and transverse positions with respect to the target nucleus center. We combine them into a single argument $z=\{v, b\}$ for nucleus $\mathrm{A}$ and $u=\{w, c\}$ for nucleus $\mathrm{B}$. The (variable) number of partons will be denoted correspondingly by $n$ and $l$. In these notations the initial state of the nucleus $\mathrm{A}$ is

$$
|A\rangle=\sum_{n=1}^{\infty} \int d \tau_{A}(n) \Psi_{A, n}\left(z_{1}, \ldots, z_{n}\right)\left|n, z_{i}\right\rangle,
$$

where $d \tau_{A}(n)=\prod_{i=1}^{n} d^{3} z_{i}$ stands for the phase space volume of the configuration. Wave functions $\Psi_{A, n}$ of the $n$-parton configuration, symmetric in their arguments, are normalized to fulfill $\langle A \mid A\rangle=1$ :

$$
W_{n} \equiv \int d \tau(n)\left|\Psi_{A, n}\left(z_{1}, \ldots, z_{n}\right)\right|^{2}, \quad \sum_{n} W_{n}=1 .
$$

We will assume a Poissonian distribution for the number of partons: $w_{n}=e^{-\langle n\rangle} / n$ ! and, as we neglect intrinsic partonic correlations, a factorization property for the wave function

$$
\left|\Psi_{A, n}\left(z_{i}\right)\right|^{2}=c_{n} \prod_{i=1}^{n} \rho_{A}\left(z_{i}\right) .
$$

The Poissonian distribution immediately implies

$$
c_{n}=\frac{1}{n !} e^{-\langle n\rangle}, \quad\langle n\rangle=\int d^{3} z \rho_{A}(z)
$$

where $\langle n\rangle$ corresponds to the average number of partons in nucleus A. The same notations are used also for nucleus B.

The paper is organized as follows. In sections [I and III we derive expressions for 2-parton inclusive cross section for two different cases. The case when both partons belong to the same forward rapidity region is examined in the section III derivation for the forward-backward case is presented in section III. The form of the two-parton cross sections implies that non-trivial forward-backward correlations emerge in high- $p_{t}$ parton production. In section $[\nabla]$ a quantity measuring the strength of correlations, 
correlation coefficient, is proposed which appears to be expressed in a simple way in terms of the derived cross sections. Numerical values for the correlation coefficient followed by discussion are presented in section $\nabla$ for light and heavy nuclei interactions at RHIC and LHC energies. The interpretation of our results and conclusions follow in section VI.

\section{TWO JETS IN THE SAME DIRECTION}

The double inclusive cross-section to produce two jets in the same (forward) direction is obtained as a direct generalization of the single inclusive cross-section in [11]. As indicated above, here we limit our studies to the production of partons at rapidities well separated from zero and neglect changes in the parton longitudinal momenta during the interaction. This implies that both observed partons originate from the same nucleus (projectile A).

We have to fix the final state $A^{\prime}$ of the nucleus A to describe the two produced partons. Since the total wave function is symmetric in all the partons we choose them to be the first and the second one and account for other possibilities by factor $\sqrt{n(n-1)}$ :

$$
\Psi_{A^{\prime}, n}\left(z_{1}, z_{2}, z_{3}, \ldots z_{n}\right)=\sqrt{n(n-1)} \psi_{\alpha_{1}}\left(z_{1}\right) \psi_{\alpha_{2}}\left(z_{2}\right) \tilde{\Psi}_{A^{\prime}, n-2}\left(z_{3}, \ldots z_{n}\right)
$$

where $\tilde{\Psi}_{A^{\prime}, n-2}\left(z_{3}, \ldots z_{n}\right)$ is the symmetrized wave function of the unobserved $n-2$ partons and $\psi_{\alpha_{i}}\left(z_{i}\right), i=1,2$ are the wave functions of the observed partons in the final state. $\alpha_{i}$ combine scaling variables and transverse momenta $p_{1}$ and $p_{2}$ of the latter.

The probability to observe the two partons is given by the modulus squared of the $S$-matrix element $\left\langle A B|S| A^{\prime} B^{\prime}\right\rangle$ summed over all possible final states of the unobserved partons. In our approximation (purely elastic collisions) the number of partons is not changed by the interaction. So the $S$-matrix is diagonal in the basis $\left\{n, z_{i} ; l, u_{i}\right\}$ :

$$
\begin{aligned}
\left\langle n^{\prime}, z_{i}^{\prime}, l^{\prime}, u_{j}^{\prime}|S| n, z_{i} ; l, u_{j}\right\rangle & \\
=\delta_{n n^{\prime}} & \delta_{l l^{\prime}} \prod_{i=1}^{n} \delta^{(3)}\left(z_{i}-z_{i}^{\prime}\right) \prod_{j=1}^{l} \delta^{(3)}\left(u_{i}-u_{i}^{\prime}\right) S_{n l}\left(z 1, \ldots, z_{n} \mid u_{1}, \ldots, u_{l}\right) .
\end{aligned}
$$

Following ref. [11] we take the square modulus of (6) making use of the specific form for the final-state wave function of the projectile nucleus (5). The double differential cross-section at a fixed overall impact parameter $\beta$ which follows reads

$$
\begin{gathered}
\frac{d \sigma_{\alpha_{1} \alpha_{2}}}{d^{2} \beta}=\sum_{n=2}^{\infty} \sum_{l \geq 0} n(n-1) \int d z_{1} d z_{2} d z_{1}^{\prime} d z_{2}^{\prime} \psi_{\alpha_{1}}\left(z_{1}^{\prime}\right) \psi_{\alpha_{2}}\left(z_{1}^{\prime}\right) \psi_{\alpha_{1}}^{*}\left(z_{1}\right) \psi_{\alpha_{2}}^{*}\left(z_{2}\right) \\
\int d \tau_{A}(n-2) d \tau_{B}(l) \Psi_{A, n}^{*}\left(z_{1}^{\prime}, z_{2}^{\prime}, z_{3}, \ldots z_{n}\right) \Psi_{A, n}\left(z_{1}, z_{2}, z_{3}, \ldots z_{n}\right)\left|\Psi_{B, l}\left(u_{1}, \ldots, u_{l}\right)\right|^{2} \\
{\left[S_{n l}\left(z_{1}, z_{2}, z_{3} \ldots z_{n} \mid u_{1}, \ldots u_{l}\right)-1\right]\left[S_{n l}^{*}\left(z_{1}^{\prime}, z_{2}^{\prime}, z_{3} \ldots z_{n} \mid u_{1}, \ldots u_{l}\right)-1\right]}
\end{gathered}
$$

The product of square brackets in (7) gives four terms. The double inclusive crosssection for transverse momenta $p_{1}, p_{2} \gg 1 / R_{A, B}$ corresponds only to the term which 
originates from the product of the $S$-matrices. Indeed in the Glauber approximation we assume that the $S$-matrix is a product of $S$-matrices for pair parton collisions. Then terms linear in $S$ or $S^{*}$ diagrammatically correspond to cutting the forward scattering amplitude either to the extreme left or to the extreme right of all partonic interactions. In both cases the momenta of intermediate partons in the cut coincide with their values in the initial colliding nuclei, so that their transverse momenta are small, of the order of typical nuclear scale $1 / R_{A, B}$ (see [11] for more detail).

We present each of the $S$-matrices as a product of the elementary partonic ones,

$$
S_{n l}\left(z_{1}, \ldots, z_{n} \mid u_{1}, \ldots, u_{l}\right)=\prod_{i=1}^{n} \prod_{j=1}^{l} s_{i j}
$$

Here $s_{i j}=1+i a\left(z_{i}, u_{j}\right)$ and $a\left(z_{i}, u_{j}\right)$ are the parton-parton scattering matrix and amplitude respectively. Since only elastic parton scattering are considered, unitarity of the partonic $s$-matrices means $s_{i j} s_{i j}^{*}=1$. So we obtain that

$$
\begin{gathered}
S_{n l}^{*}\left(z_{1}^{\prime}, z_{2}^{\prime}, z_{3} \ldots z_{n} \mid u_{1}, \ldots u_{l}\right) S_{n l}\left(z_{1}, z_{2}, z_{3} \ldots z_{n} \mid u_{1}, \ldots u_{l}\right) \\
=\prod_{i=1,2} \prod_{j=1}^{l}\left[1+i a\left(z_{i}^{\prime}, u_{j}\right)\right]^{*}\left[1+i a\left(z_{i}, u_{j}\right)\right] .
\end{gathered}
$$

and does not depend on $z_{3}, \ldots z_{n}$. This allows to integrate over these variables and sum over $n$ to produce the density matrix $\rho_{A}$ of the nucleus A for a pair of partons:

$$
\sum_{n} n(n-1) \int d \tau_{A}(n-2) \Psi_{A, n}^{*}\left(z_{1}^{\prime}, z_{2}^{\prime}, z_{3}, \ldots z_{n}\right) \Psi_{A, n}\left(z_{1}, z_{2}, z_{3}, \ldots z_{n}\right)=\rho_{A}\left(z_{1}, z_{2} \mid z_{1}^{\prime}, z_{2}^{\prime}\right)
$$

Factor $n(n-1)$ again accounts the possibility that any two of the partons entering the symmetric wave function can be chosen. Thus we get

$$
\begin{gathered}
\frac{d \sigma_{\alpha_{1} \alpha_{2}}}{d^{2} \beta}=\sum_{l} \int d z_{1} d z_{2} d z_{1}^{\prime} d z_{2}^{\prime} \psi_{\alpha_{1}}\left(z_{1}^{\prime}\right) \psi_{\alpha_{2}}\left(z_{1}^{\prime}\right) \psi_{\alpha_{1}}^{*}\left(z_{1}\right) \psi_{\alpha_{2}}^{*}\left(z_{2}\right) \rho_{A}\left(z_{1}, z_{2} \mid z_{1}^{\prime}, z_{2}^{\prime}\right) \\
\int d \tau_{B}(l)\left|\Psi_{B, l}\left(u_{j}\right)\right|^{2}\left\{\prod_{i=1,2} \prod_{j=1}^{l}\left[1+i a\left(z_{i}^{\prime}, u_{j}\right)\right]^{*}\left[1+i a\left(z_{i}, u_{j}\right)\right]-1\right\} .
\end{gathered}
$$

Making use of the factorization of the wave function (3) we have

$$
\rho_{A}\left(z_{1}, z_{2} \mid z_{1}^{\prime}, z_{2}^{\prime}\right)=\rho_{A}\left(z_{1} \mid z_{1}^{\prime}\right) \rho_{A}\left(z_{2} \mid z_{2}^{\prime}\right) \quad \text { and } \quad\left|\Psi_{B, l}\left(u_{j}\right)\right|^{2}=\frac{1}{l !} e^{-\langle l\rangle} \prod_{j=1}^{l} \rho_{B}\left(u_{j} \mid u_{j}\right) .
$$

Assuming factorization of partonic transverse and longitudinal degrees of freedom, for equal scaling variables $v_{i}=v_{i}^{\prime}=v$, we have

$$
\rho_{A}\left(v, b_{i} \mid b_{i}^{\prime}\right)=P_{A}(v) \tilde{\rho}_{A}\left(b_{i} \mid b_{i}^{\prime}\right),
$$

where $P_{A}(v)$ is the mean parton number distribution and $\tilde{\rho}_{A}\left(b_{i} \mid b_{i}^{\prime}\right)$ is the transverse part of single parton density matrix. For equal arguments it goes into the standard 



Assuming for simplicity a central collision (in other case the argument in all $T_{A}$ 's must be shifted by the impact parameter $\beta$ ) we get

$$
\begin{gathered}
I_{A B}\left(\beta, y_{1}, p, y_{2}, q\right)=\sum_{n l \geq 1} n l \int d z_{1} d z_{1}^{\prime} d u_{1} d u_{1}^{\prime} \psi_{p}\left(z_{1}^{\prime}\right) \psi_{q}\left(u_{1}^{\prime}\right) \psi_{p}^{*}\left(z_{1}\right) \psi_{q}^{*}\left(u_{1}\right) \rho_{A}\left(z_{1} \mid z_{1}^{\prime}\right) \rho_{B}\left(u_{1} \mid u_{1}^{\prime}\right) \\
\left\{\left[1+i a\left(z_{1}^{\prime}, u_{1}^{\prime}\right)\right]^{*}\left[1+i a\left(z_{1}, u_{1}\right)\right]\left(\prod_{j=2}^{l} \int d u_{j} \rho_{B}\left(u_{j} \mid u_{j}\right)\left[1+i a\left(z_{1}^{\prime}, u_{j}\right)\right]^{*}\left[1+i a\left(z_{1}, u_{j}\right)\right]\right)\right. \\
\left.\left(\prod_{i=2}^{n} \int d z_{i} \rho_{A}\left(z_{i} \mid z_{i}\right)\left[1+i a\left(z_{i}, u_{1}^{\prime}\right)\right]^{*}\left[1+i a\left(z_{i}, u_{1}\right)\right]\right)-\int \prod \rho_{B} \prod \rho_{A}\right\} \frac{e^{-\langle n\rangle-\langle l\rangle}}{l ! n !}(28)
\end{gathered}
$$

Already at this stage it is convenient to separate from the total inclusive crosssection its part which does not contain interactions between the observed partons, that is the term which comes with unity from the product of the first two square brackets in (28). Comparing with [1]] we find it to be:

$$
\begin{gathered}
I_{A B}^{(1)}\left(\beta, y_{1}, p, y_{2}, q\right)=\left\{\sum_{n \geq 1} \frac{e^{-\langle n\rangle}}{(n-1) !} \int d z_{1} d z_{1}^{\prime} \psi_{p}\left(z_{1}^{\prime}\right) \psi_{p}^{*}\left(z_{1}\right) \rho_{A}\left(z_{1} \mid z_{1}^{\prime}\right)\right. \\
\left.\left(\prod_{j=2}^{l} \int d u_{j} \rho_{B}\left(u_{j} \mid u_{j}\right)\left[1+i a\left(z_{1}^{\prime}, u_{j}\right)\right]^{*}\left[1+i a\left(z_{1}, u_{j}\right)\right]-\int \prod_{j=2}^{l} d u_{j} \rho_{B}\left(u_{j} \mid u_{j}\right)\right)\right\} \\
\left\{\sum_{l \geq 1} \frac{e^{-\langle l\rangle}}{(l-1) !} \int d u_{1} d u_{1}^{\prime} \psi_{q}^{*}\left(u_{1}\right) \psi_{q}\left(u_{1}^{\prime}\right) \rho_{B}\left(u_{1} \mid u_{1}^{\prime}\right)\right. \\
\left.\left(\prod_{i=2}^{n} \int d z_{i} \rho_{A}\left(z_{i} \mid z_{i}\right)\left[1+i a\left(z_{i}, u_{1}^{\prime}\right)\right]^{*}\left[1+i a\left(z_{i}, u_{1}\right)\right]-\int \prod_{i=2}^{n} d z_{i} \rho_{A}\left(z_{i} \mid z_{i}\right)\right)\right\} \\
=I_{A}\left(y_{1}, p\right) I_{B}\left(y_{2}, q\right)
\end{gathered}
$$

where $I_{A(B)}$ is the inclusive cross-section to produce a single jet from nucleus $A(B)$ in AB collisions at impact parameter $\beta$ (see (20)). So this term factorizes into a product of two independent single inclusive cross-sections to produce each of the observed partons. It is important that this result is exact and not based on the smallness of the parton interaction range on the nuclear scale. In particular, its validity is not spoiled by corrections of the order $1 / A^{2 / 3}$.

Now we turn to the rest terms in (28). In them the integrals in round brackets can be done exactly as in the standard Glauber derivation, taking into account that the space range of the partonic interaction is much smaller than the nuclear scale (see [11]). After that summations in $n$ and $l$ can be easily performed and we get

$$
\begin{aligned}
& I_{A B}^{(2)}\left(\beta, y_{1}, p, y_{2}, q\right)=\int d^{2} b_{1} d^{2} b_{1}^{\prime} d^{2} c_{1} d^{2} c_{1}^{\prime} \\
& e^{i p\left(b_{1}-b_{1}^{\prime}\right)+i q\left(c_{1}-c_{1}^{\prime}\right)} \rho_{A}\left(v, b_{1} \mid b_{1}^{\prime}\right) \rho_{B}\left(w, c_{1} \mid c_{1}^{\prime}\right)
\end{aligned}
$$




$$
\left\{\left(\left[1+i a\left(b_{1}^{\prime}-c_{1}^{\prime}, v, w\right)\right]^{*}\left[1+i a\left(b_{1}-c_{1}, v, w\right)\right]-1\right) e^{E_{B}\left(v, b_{1}, b_{1}^{\prime}\right)+E_{A}\left(w, c_{1}, c_{1}^{\prime}\right)}-1\right\}
$$

where

$$
E_{B}\left(v, b_{1}, b_{1}^{\prime},\right)=T_{B}\left(\left(b_{1}+b_{1}^{\prime}\right) / 2\right)\left(F_{B}\left(v, b_{1}-b_{1}^{\prime}\right)-F_{B}(v, 0)\right)
$$

$E_{A}$ is defined by a similar formula for nucleus $\mathrm{A}$ and $y_{1}, y_{2}, p_{1}, p_{2}$ are the rapidities and transverse momenta of the observed particles(partons), and $v, w$ are longitudinal momentum fractions corresponding to $y_{1}$ and $y_{2}$ respectively. Functions $F_{B}(v, b)$ is the Fourier transform of the transverse momentum distributions $I(v, w, p)$ in the elastic scattering of two partons with scaling variable $v$ and $w$, averaged over the longitudinal momenta of nucleus B partons (see [11]):

$$
F_{B}(v, b)=\int d w P(w) \int \frac{d^{2} p}{(2 \pi)^{2}} I(p, v, w) e^{i p b}
$$

It is convenient to introduce new variables for integration. Define

$$
r_{1} \equiv b_{1}-b_{1}^{\prime} ; r_{2} \equiv c_{1}-c_{1}^{\prime} ; b \equiv\left(b_{1}+b_{1}^{\prime}\right) / 2 ; \quad c \equiv\left(c_{1}+c_{1}^{\prime}\right) / 2
$$

In these variables the cross section is rewritten as

$$
\begin{gathered}
I_{A B}^{(2)}\left(\beta, y_{1}, p, y_{2}, q\right)=\int d^{2} r_{1} d^{2} r_{2} d^{2} b d^{2} c e^{i p r_{1}+i q r_{2}} \rho_{A}\left(v, b_{1} \mid b_{1}^{\prime}\right) \rho_{B}\left(w, c_{1} \mid c_{1}^{\prime}\right) \\
\left\{\left(\left[1+i a\left(b-c-\frac{r_{1}-r_{2}}{2}, v, w\right)\right]^{*}\left[1+i a\left(b-c+\frac{r_{1}-r_{2}}{2}, v, w\right)\right]-1\right) e^{E_{B}\left(v, b, r_{1}\right)+E_{A}\left(w, c, r_{2}\right)}-1\right\}
\end{gathered}
$$

where in the new variables

$$
E_{B}\left(v, b, r_{1}\right)=T_{B}(b)\left(F_{B}\left(v, r_{1}\right)-F_{B}(v, 0)\right)
$$

and similarly for $E_{A}$.

Assuming that $E(. . b, c .$.$) and density matrices change significantly only when b$ and $c$ suffer macroscopic shifts of about nucleus radius, we can perform integrations in $c$. Take the terms in (20) containing single amplitudes $i a\left(b-c \pm\left(r_{1}-r_{2}\right) / 2\right)$. On the nuclear scale they can be effectively substituted as

$$
i a\left(b-c \pm\left(r_{1}-r_{2}\right) / 2\right) \rightarrow i \tilde{a}(0) \delta^{2}\left(b-c \pm\left(r_{1}-r_{2}\right) / 2\right) \simeq i \tilde{a}(0) \delta^{2}(b-c)
$$

where $\tilde{a}$ is the amplitude in the transverse momentum space and we have used that $\left(r_{1}-r_{2}\right) / 2$ is small on the nuclear scale. In the term with the product

$$
a\left(b-c+\left(r_{1}-r_{2}\right) / 2\right) a^{*}\left(b-c-\left(r_{1}-r_{2}\right) / 2\right)
$$

we pass from the integration variable $c$ to $r=b-c+\left(r_{1}-r_{2}\right) / 2$ in which this product takes the form

$$
a(r) a^{*}\left(r-r_{1}+r_{2}\right)
$$



where we defined the single cross-section at fixed $b$ as the integrand in (20) (for the central collision, $\beta=0$ )

$$
I_{A}(p, v, b)=P_{A}(v) T_{A}(b) \int d^{2} r e^{i p r}\left(e^{T_{B}(b)\left(F_{b}(v, r)-F_{B}(v, 0)\right)}-e^{-T_{B}(b) F_{B}(v, 0)}\right)
$$

The third term corresponds to $v, p \leftrightarrow w, q$

$$
I_{A B}^{(23)}=(2 \pi)^{2} P_{A}(v) \frac{d \sigma(p, v, w)}{d^{2} p} \int d^{2} b T_{A}(b) e^{-T_{B}(b) F_{B}(v, 0)} I_{B}(p+q, w, b)
$$

Finally in terms of $I_{A,(B)}(p+q, v(w), b)$

$$
\begin{gathered}
I_{A B}^{(21)}=\int d^{2} b\left(\int d^{2} l \frac{d \sigma(l, v, w)}{d^{2} l} I_{A}(p+l, v, b) I_{B}(q-l, w, b)\right. \\
\left.-\sigma^{t o t}(v, w) I_{A}(p, v, b) I_{B}(q, w, b)\right)
\end{gathered}
$$

\section{CORRELATIONS}

We restrict ourselves to the study of the forward-backward multiplicity correlations. Since the correlations are in any case small we may restrict ourselves to a linear dependence of the average multiplicity in the backward window at a fixed multiplicity in the forward window as a function of the latter;

$$
\frac{\left\langle n_{B}\right\rangle_{n_{F}}}{\left\langle n_{B}\right\rangle}\left(n_{F}\right)=a+b n_{F}
$$

Here $\left\langle n_{B}\right\rangle$ is the overall average of the multiplicity in the backward window (at all $n_{F}$ ). Thus defined coefficient $b$ shows the relative deviation of the conditional average $\left\langle n_{B}\right\rangle_{n_{F}}$ when the number of jets observed in the forward rapidity window $n_{F}$ changes by unity. It can be expressed via averages of linear and bilinear products of multiplicities:

$$
b=\frac{1}{\left\langle n_{B}\right\rangle} \frac{\left\langle n_{B} n_{F}\right\rangle-\left\langle n_{B}\right\rangle\left\langle n_{F}\right\rangle}{\left\langle n_{F}^{2}\right\rangle-\left\langle n_{F}\right\rangle^{2}}
$$

One gets this expression by multiplying (42) first by $p\left(n_{F}\right)$ then by $n_{F} p\left(n_{F}\right)$, summing over $n_{F}$ and solving the arising system of linear equations for $a$ and $b$.

To compute the mentioned bilinear products we first point out that $n$ ! in the denominator in the phase space volume for $n$ identical particles should refer only to particles within the same phase space volume. For our problem it implies that identical particles produced in different rapidity windows can be considered as different (see Appendix for details). This allows to immediately obtain simple expressions for the squares of multiplicities coming from independent pair production described by the product of single inclusive cross-sections. Consider first emission of two particles into the forward rapidity window. At a fixed overall impact parameter $\beta$ we find

$$
\left\langle n_{F}\right\rangle=c \int \frac{d y d^{2} p}{(2 \pi)^{2}} I_{A}(y, p)
$$


and

$$
\left\langle n_{F}\left(n_{F}-1\right)\right\rangle=c \int \frac{d y_{1} d^{2} p_{1} d y_{2} d^{2} p_{2}}{(2 \pi)^{4}} I_{A A}\left(\beta, y_{1}, p_{1}, y_{2}, p_{2}\right)
$$

where integrations over $y$ are restricted to the forward rapidity window and $c=$ $1 /\left(d^{2} \sigma_{A B}(\beta) / d^{2} \beta\right)$ where $d^{2} \sigma_{A B}(\beta) / d^{2} \beta$ is the AB inelastic cross-section at fixed impact parameter $\beta$. The latter is practically unity for $\beta<R_{A}+R_{B}$. As shown in section [I] (Eq. (19) ), in the first approximation, the double inclusive cross-section in (45) is just a product of two single inclusive ones. This gives a relation

$$
\left\langle n_{F}\left(n_{F}-1\right)\right\rangle=\frac{1}{c}\left\langle n_{F}\right\rangle^{2}
$$

which, with $c \simeq 1$ leads to

$$
\left\langle n_{F}^{2}\right\rangle-\left\langle n_{F}\right\rangle^{2}=\left\langle n_{F}\right\rangle
$$

(effectively the distribution seems to be Poissonian). Note that the dispersion is different from zero. This is the reason why for the production of the pair into the same (forward) rapidity window we can limit to the first approximation in powers of $1 / A$ or $1 / B$.

Passing to the emission of jets into different rapidity windows, we may consider the jets different. So instead of (45) we shall find

$$
\left\langle n_{F} n_{B}\right\rangle=c \int \frac{d y_{1} d^{2} p d y_{2} d^{2} q}{(2 \pi)^{4}} I_{A B}\left(\beta, y_{1}, p, y_{2}, q\right)
$$

From independent production (part $I_{A B}^{(1)}$ ) we shall get just the product of average multiplicities, so that

$$
\left\langle n_{F} n_{B}\right\rangle-\left\langle n_{F}\right\rangle\left\langle n_{B}\right\rangle=\int \frac{d y_{1} d^{2} p d y_{2} d^{2} q}{(2 \pi)^{4}} I_{A B}^{(2)}\left(\beta, y_{1}, p, y_{2}, q\right)
$$

where we used $c \simeq 1$.

This gives for the correlation coefficient $b$

$$
b=\frac{\int d y_{1} d^{2} p d y_{2} d^{2} q I_{A B}^{(2)}\left(\beta, y_{1}, p, y_{2}, q\right)}{\int d y_{1} d^{2} p I_{A}\left(y_{1}, p\right) \int d y_{2} d^{2} q I_{A}\left(y_{2}, q\right)}
$$

where integrals over $y_{1}, p$ and $y_{2}, q$ go over the forward and backward rapidity windows, respectively. We see that in the numerator of the expression for the correlation coefficient the leading terms in powers of $1 / A$ and $1 / B$ cancel and only subleading terms of the relative order $1 / A^{2 / 3}$ or $1 / B^{2 / 3}$ remain. This means that in any case forwardbackward multiplicity correlations at a fixed $\beta$ have the order $1 / A^{2 / 3}$ or $1 / B^{2 / 3}$. So for their observation collision of comparatively light nuclei is preferable. To calculate the correlations one has to evaluate the integrals in (50).

Our definition of the correlation coefficient differs from the conventional one which reads $b_{0}=\operatorname{Cov}\left(n_{B}, n_{F}\right) / \sqrt{D\left(n_{B}\right) D\left(n_{F}\right)}$ and for equal dispersions of forward and backward multiplicities is $b_{0}=\left(\left\langle n_{F} n_{B}\right\rangle-\left\langle n_{F}\right\rangle\left\langle n_{B}\right\rangle\right) /\left(\left\langle n_{F}^{2}\right\rangle-\left\langle n_{F}\right\rangle^{2}\right)$. As one can see comparing $b_{0}$ to (43) the difference is by factor $\left\langle n_{B}^{2}\right\rangle-\left\langle n_{B}\right\rangle\left\langle n_{B}\right\rangle$. As this factor is proportional to the backward rapidity window width $\Delta y_{2}$ (see (47)), the correlation coefficient (43) has an advantage compared to $b_{0}$ having no explicit dependence on the chosen rapidity intervals. However the value of correlation coefficient $b$ is not limited from above by unity as for $b_{0}$. 


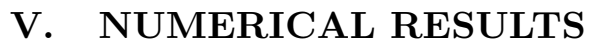

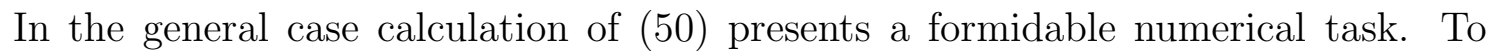

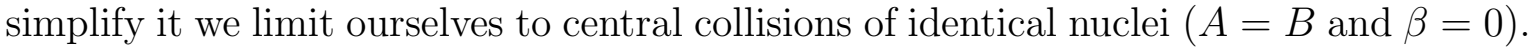

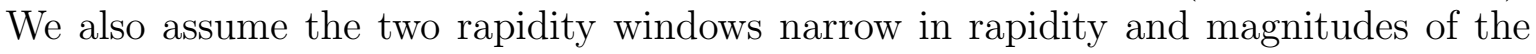

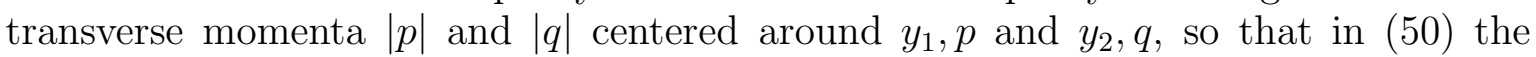

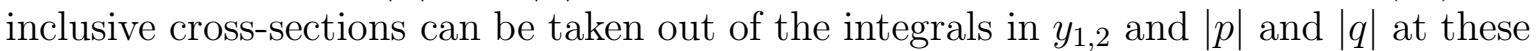

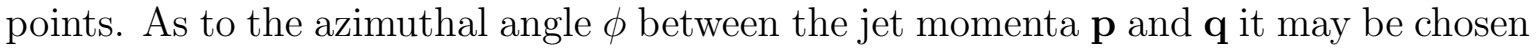

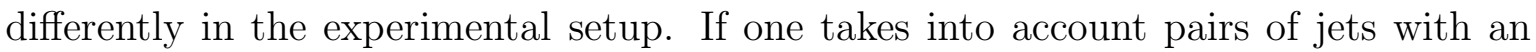

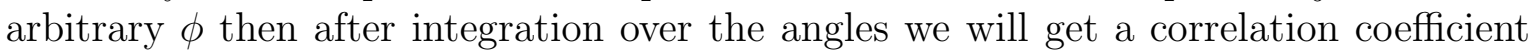

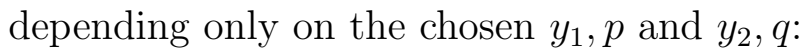

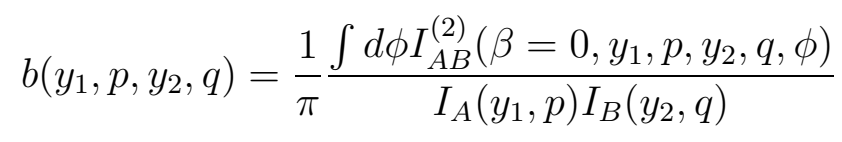

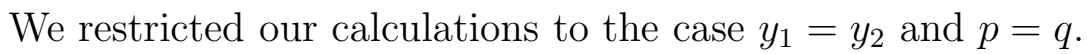

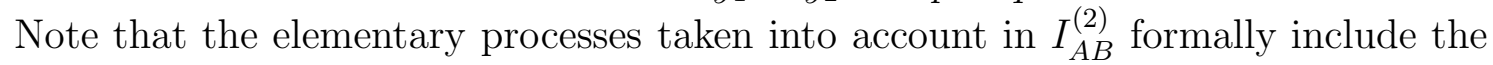

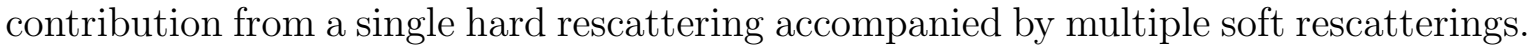

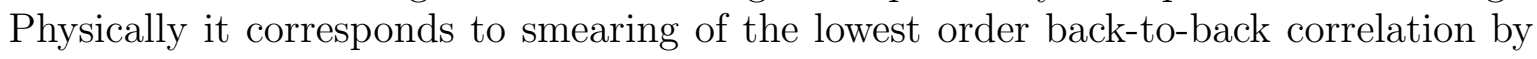

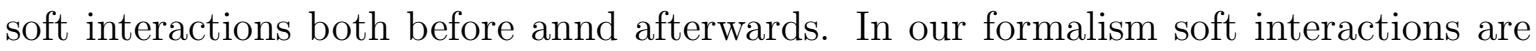

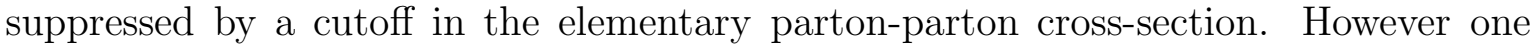

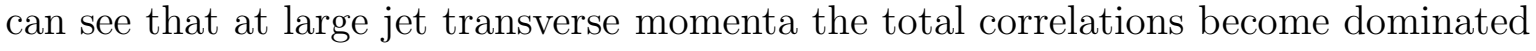

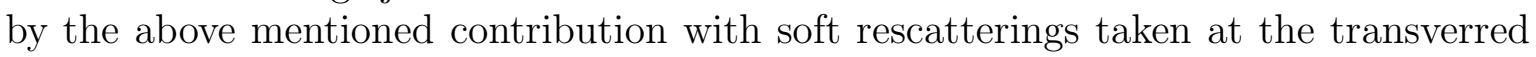

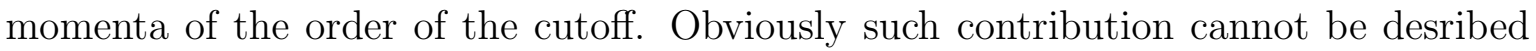

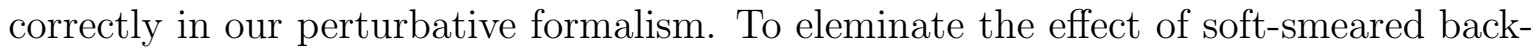

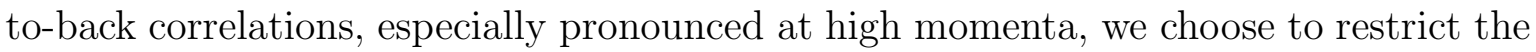

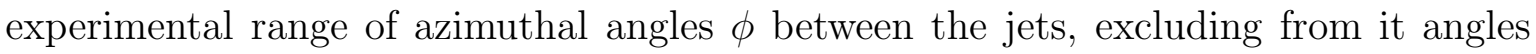

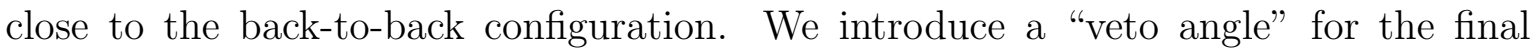

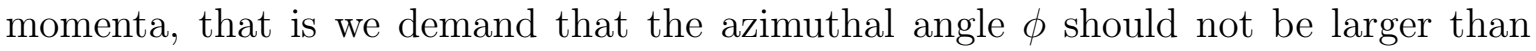

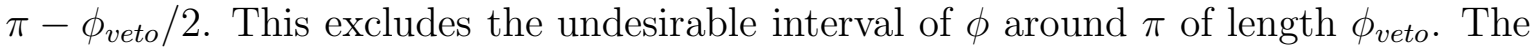

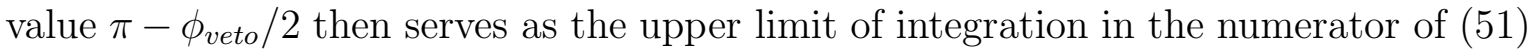

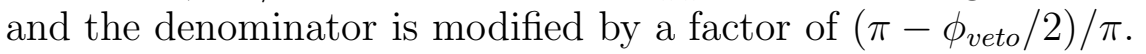

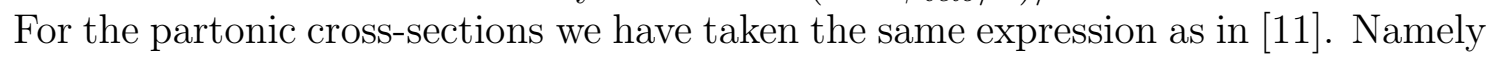

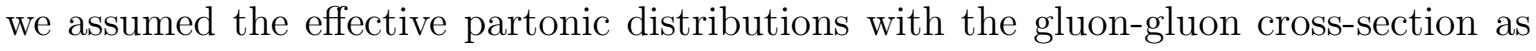

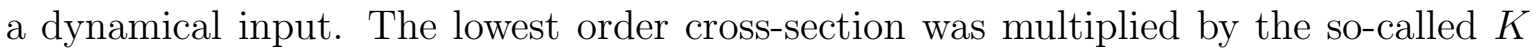

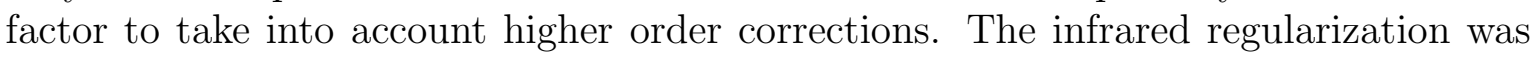

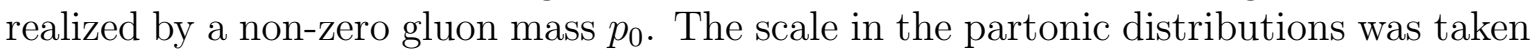

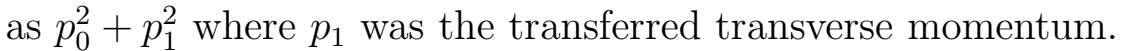

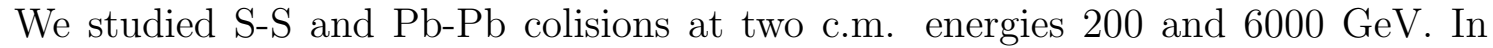

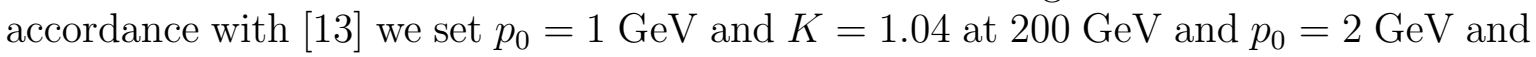

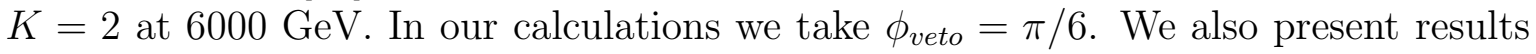

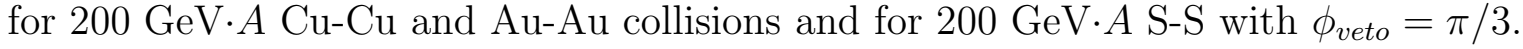

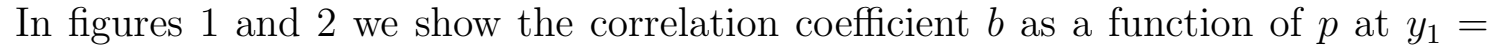

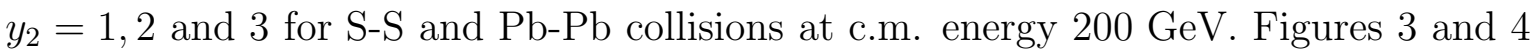

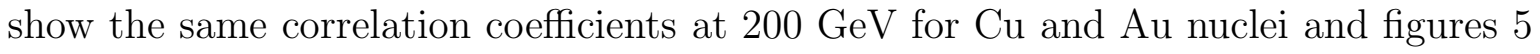

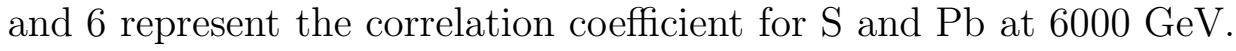

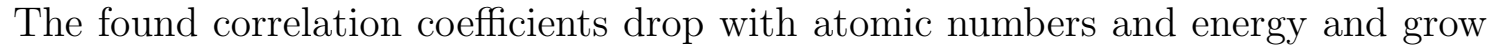

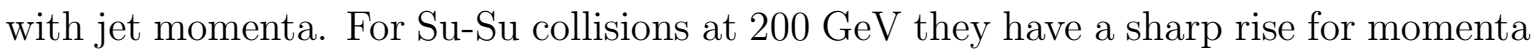

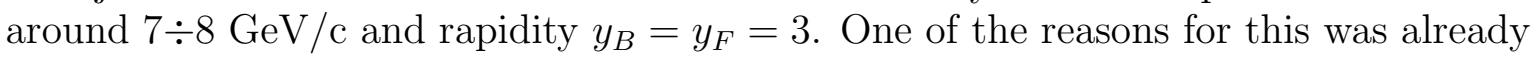




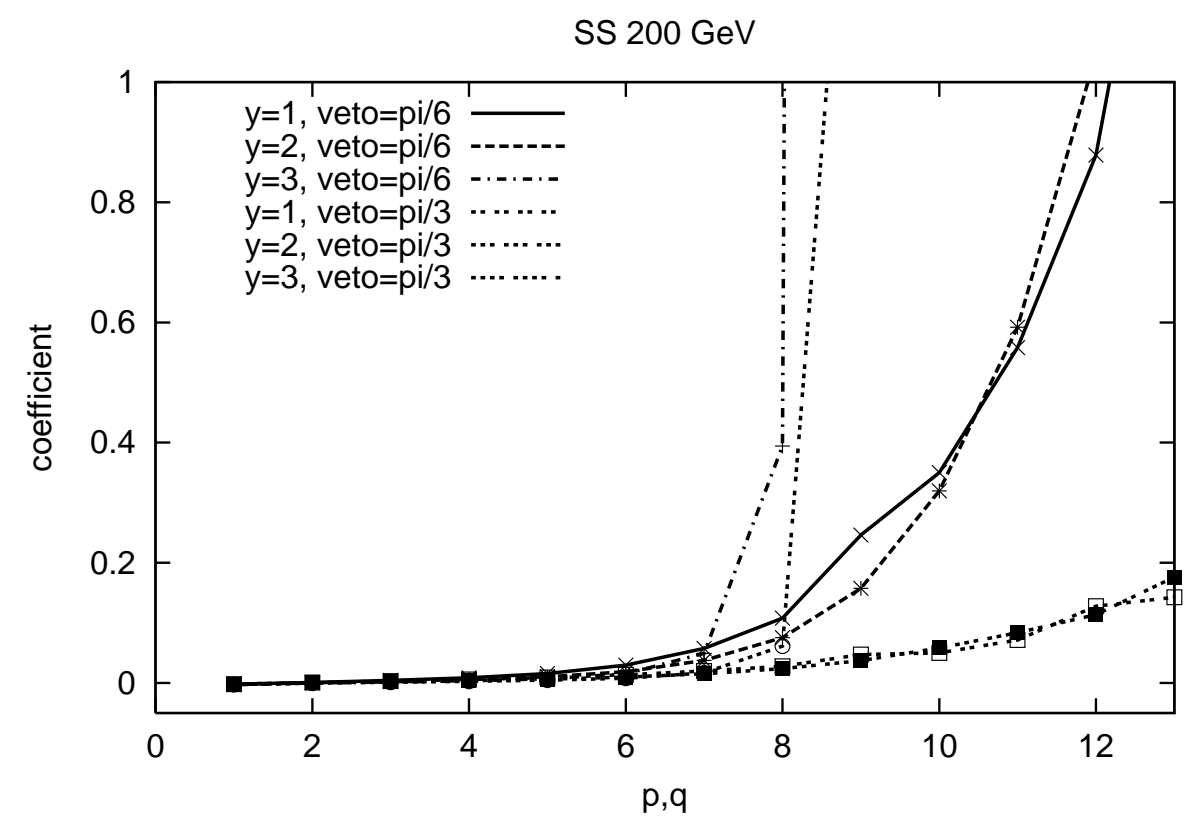

FIG. 1: Correlation coefficient for sulphur-sulphur collisions at $\sqrt{s}=200 \mathrm{GeV}$.

$\mathrm{Pb}, 200 \mathrm{GeV}$

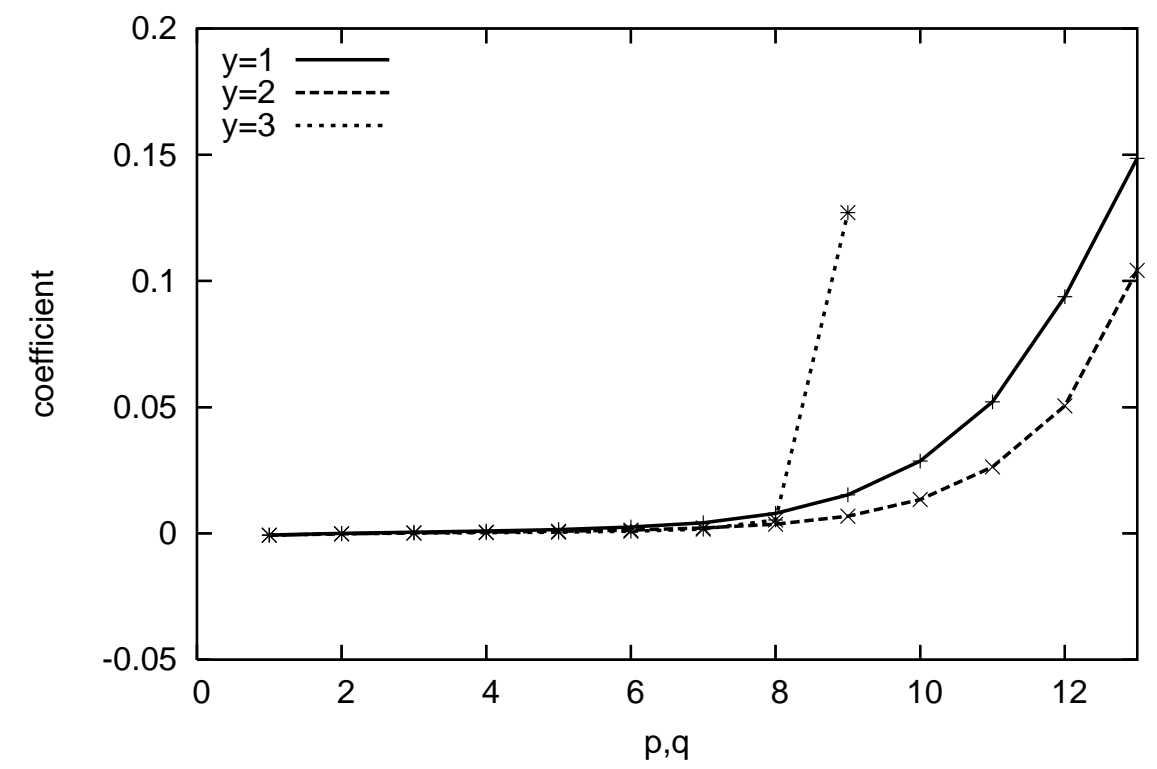

FIG. 2: Correlation coefficient for lead-lead collisions at $\sqrt{s}=200 \mathrm{GeV}$.

discussed in this section, another is due to to kinematics: the single inclusive crosssections in the denominator of (43) tend to zero at the limits of the phase space volume. As expected, for $\mathrm{Pb}-\mathrm{Pb}$ collisions the correlation coefficients are an order of magnitude smaller than for S-S collisions. 


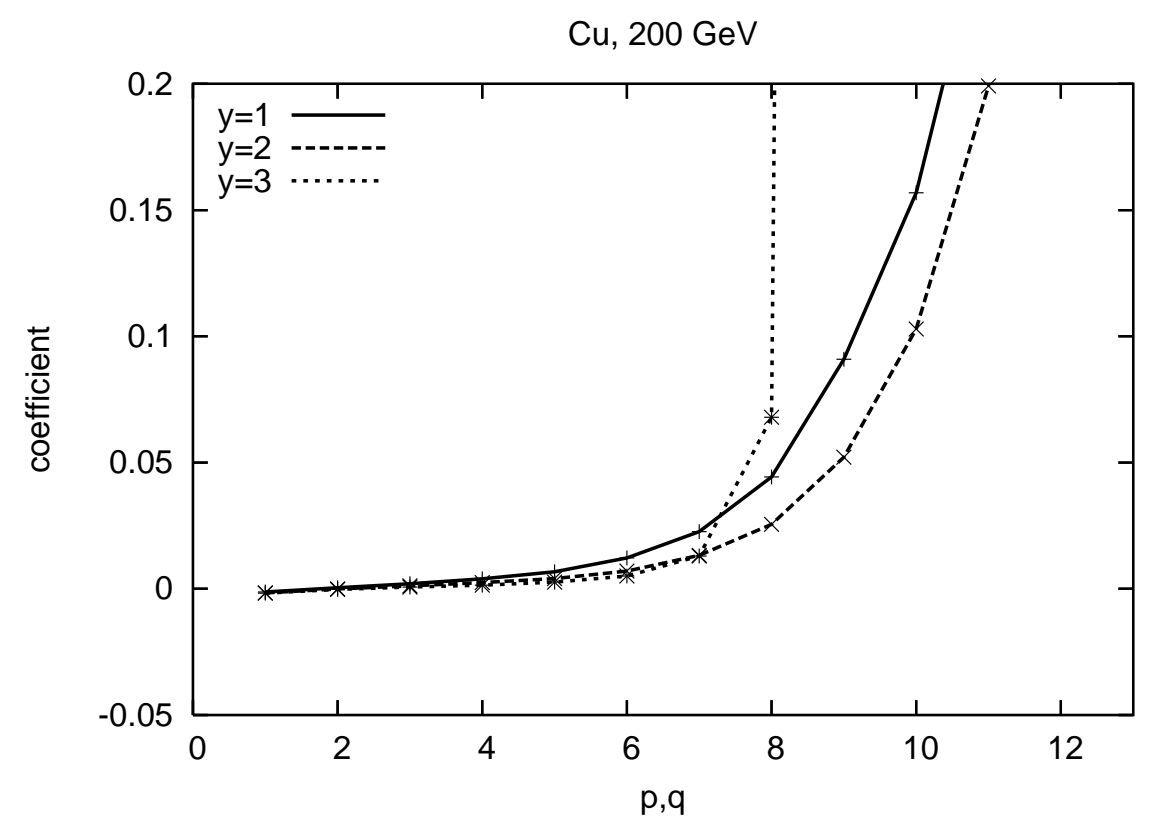

FIG. 3: Correlation coefficient for copper-copper collisions at $\sqrt{s}=200 \mathrm{GeV}$.

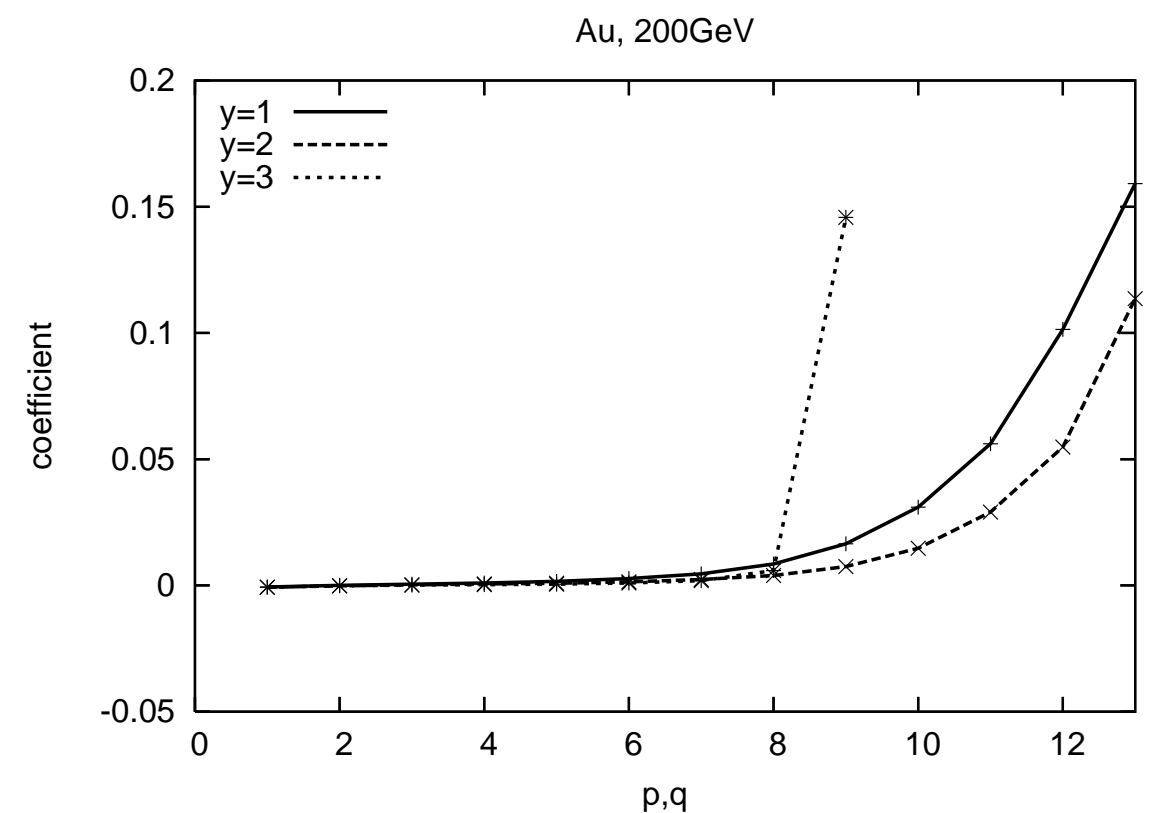

FIG. 4: Correlation coefficient for gold-gold collisions at $\sqrt{s}=200 \mathrm{GeV}$.

\section{CONCLUSIONS}

Our calculations show that in spite of the fact that in heavy nuclei long range multiplicity correlations between jets are small, of the order $A^{-2 / 3}$ for identical nuclei, they are of observable magnitude if nuclei are not too heavy. They also visibly grow with the transverse momentum and for S-S collisions at $200 \mathrm{GeV}$. For transverse momenta of jets of about $10 \div 12 \mathrm{GeV} / c$ they stay large $(10 \div 20 \%)$ even if the back-to back correlations are totally excluded by $\pi / 3$ veto angle. Their observation and mea- 


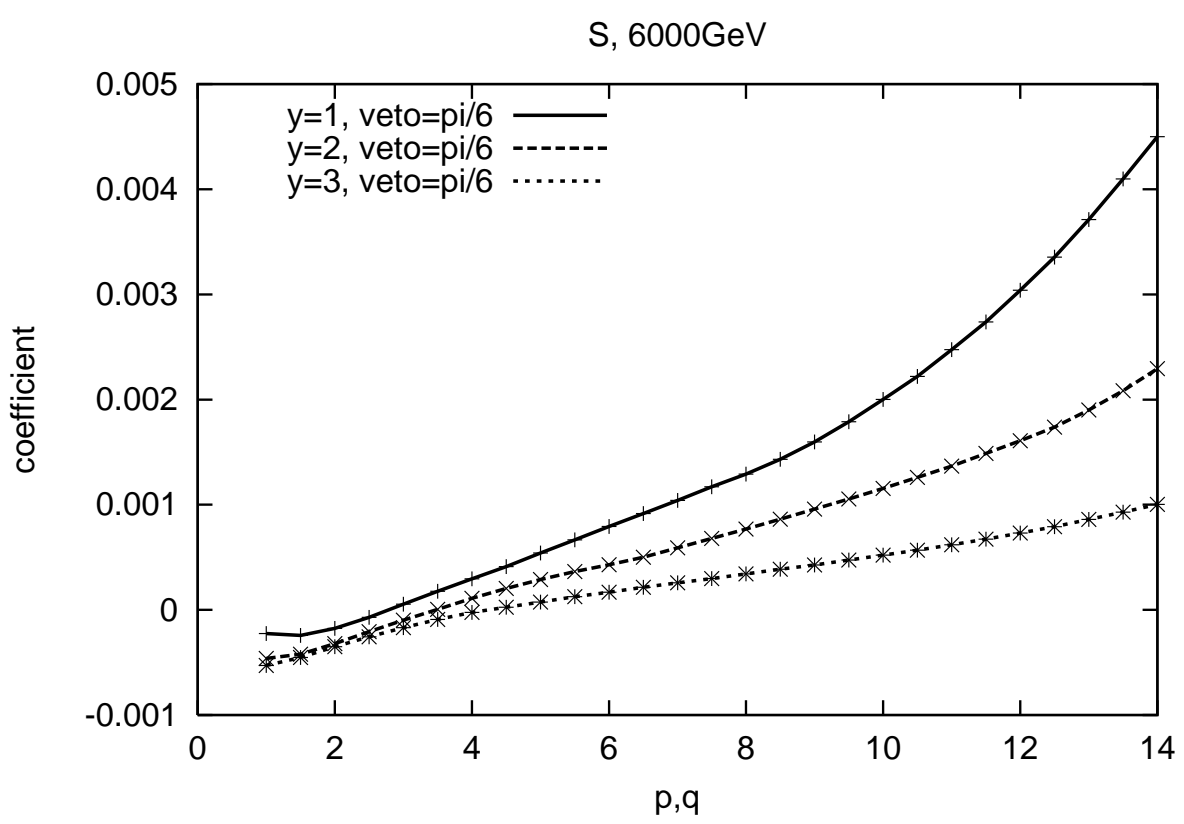

FIG. 5: Correlation coefficient for sulphur, $\sqrt{s}=6000 \mathrm{GeV}$.

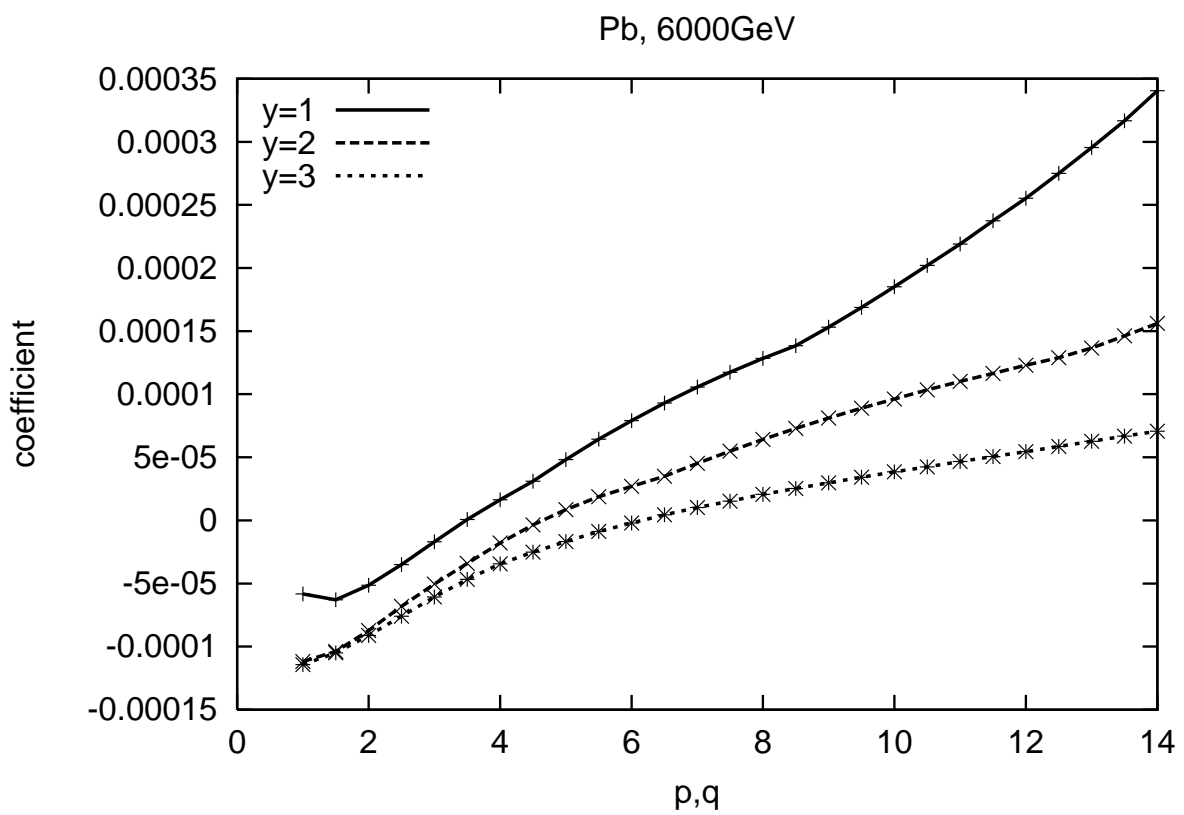

FIG. 6: Correlation coefficient for lead, $\sqrt{s}=6000 \mathrm{GeV}$.

surement for non-zero veto angles will favor a hypothesis that multiple hard collisions indeed occur before the fragmentation of jets into hadrons and are described by the perturbative QCD mechanism. However at the supposed LHC energies of $6 \mathrm{TeV}$ these correlations are strongly suppressed reaching $0.5 \%$ for S-S collisions at jet momenta of about $15 \mathrm{GeV} / c$.

Of course we understand that other effects may somewhat change our predictions based on the simple Glauber approach. Among them the most prominent is quenching of jets as they propagate through the nuclear medium. Also if the jet energy hap- 
pens to be insufficiently high the formation length may shorten to allow for the jets hadronization inside the nucleus, which will spoil our simple picture of hard rescattering. Another important point is the jet fragmentation which in general can lead to correlation pattern for hadrons different form that for partons. These questions are under our consideration at present. In any case these other effects will produce certain corrections to the results presented in this paper, which thus may serve as a natural starting point.

\section{ACKNOWLEDGEMENTS}

The authors are grateful to Dr. V.M. Suslov for his advice in the computational techinques. M.A.B. is thankful to the North Carolina Central University for hospitality and support. This work was supported by grants of Education Ministry of Russia RNP 2.1.1.1112 and RFFI of Russia 06-02-16115a. The work of R.S.K. was supported by INTAS Nr. 05-112-5031.

\section{APPENDIX. IDENTICAL PARTICLES IN DIFFERENT PARTS OF THE PHASE VOLUME (RELATED TO EQ. (47)}

Consider two particles characterized, say, by their rapidities only $y_{1}$ and $y_{2}$ which take values in the interval $[0,1]$. The integrals over intermediate state of two identical particles, between which no distinction is made, in general have the form:

$$
I=\int_{0}^{1} \frac{d y_{1} d y_{2}}{2 !} f\left(y_{1}, y_{2}\right)
$$

where $f\left(y_{1}, y_{2}\right)$ is some function symmetric in $y_{1}$ and $y_{2}$. Now we split the single particle phase volume in two, say $[0,1]=[0,1 / 2]+[1 / 2,1]$ and assume that particle with its rapidity in $[0,1 / 2]$ is particle 1 and that with its rapidity in $[1 / 2,1]$ is particle 2 . Now instead of a single intermediate state we have 3 different ones: with two particles 1 (state A), with two particles 2 (state B) and with one particle 1 and one 2 (state C). The same integral will now be given by a sum

$I_{A}+I_{B}+I_{C}=\int_{0}^{1 / 2} \frac{d y_{1} d y_{2}}{2 !} f\left(y_{1}, y_{2}\right)+\int_{1 / 2}^{1} \frac{d y_{1} d y_{2}}{2 !} f\left(y_{1}, y_{2}\right)+\int_{0}^{1 / 2} d y_{1} \int_{1 / 2}^{1} d y_{2} f\left(y_{1}, y_{2}\right)$

As we see this sum is equal to $I$ exactly. So considering particles in $[0,1 / 2]$ and $[1 / 2,1]$ as different gives the correct result.

[1] J. Adams et al, Nucl. Phys. A 757 (2005) 102; K. Adcox et at, Nucl. Phys. A 757 (2005) 184.

[2] M. Gaździcki et al, J. Phys. G 30 (2004) 701.

[3] R.Baier, D.Schiff and B.G.Zakharov, Ann. Rev. Nucl. Part. Sci 50 (2000) 37.

[4] C.A. Salgado, Mod. Phys. Lett A 19 (2004) 271.

[5] J.W. Cronin et al, Phys. Rev. D 11 (1975) 3105. 
[6] X.N. Wang, Phys. Rev. C 61 (2000) 064910.

[7] B.Z. Kopeliovich, J. Nemchik, A. Schaefer, A.V. Tarasov, Phys. Rev. Lett. 882002 232303.

[8] M. Lev, B. Petersson, Z. Phys. C21 (1983) 155.

[9] G.Calucci and D.Treleani, Phys.Rev. D 41 (1990) 3367; D 44 (1991) 2746.

[10] A.Accardi and D.Treleani, Phys. Rev. D 64 (2001) 116004.

[11] M.A.Braun, E.G.Fereiro, C.Pajares, D.Treleani, Nucl. Phys. A 723 (2003) 249.

[12] A. Accardi, M. Gyulassy, Phys. Lett. B 586 (2004) 244.

[13] E.Cataruzza and D. Treleani, Phys. Rev. D 69 (2004) 094006 (hep-ph/0401067).

[14] N.Armesto, L.McLerran and C.Pajares, hep-ph/0607345 